\title{
The Discovery of the Periodic Table : the Chemical Revolution from Lavoisier to Mendeleev
}

\author{
by Rochelle Forrester \\ Copyright (C) 2019 Rochelle Forrester \\ All Rights Reserved \\ The moral right of the author has been asserted
}

Anyone may reproduce all or any part of this paper without the permission of the author so long as a full acknowledgement of the source of the reproduced material is made.

Second Edition

Published 30 September 2019

Preface

This paper was written in order to examine the order of discovery of significant developments in the history of humankind. It is part of my efforts to put the study of social and cultural history and social change on a scientific basis capable of rational analysis and understanding. This has resulted in a hard copy book How Change Happens: A Theory of Philosophy of History, Social Change and Cultural Evolution and a website How Change Happens Rochelle Forrester's Social Change, Cultural Evolution and Philosophy of History website. There are also philosophy of history papers such as The Course of History, The Scientific Study of History, Guttman Scale Analysis and its use to explain Cultural Evolution and Social Change and the Philosophy of History and papers on Academia.edu, Figshare, Humanities Common, Open Science Framework, Orcid, Phil Papers, SocArXiv, Social Science Research Network, Vixra and Zenodo websites.

This paper is part of a series on the History of Science and Technology. Other papers in the series are

The Invention of Stone Tools Fire The Neolithic Revolution The Invention of Pottery History of Metallurgy The Development of Agriculture and Pastoralism $\quad$ History of Writing

The Invention of Glass History of Astronomy Invention of Microscopes and Telescopes

History of Printing The Invention of the Steam Engine History of Electricity

Electric Telegraph Telephone $\underline{\text { Radio }} \underline{\text { Television }}$ Photography $\underline{\text { Motion Pictures }}$

Internal Combustion Engine Motor Car Aeroplanes The History of Medicine

The Discovery of the Periodic Table The Discovery of the Atomic World

Other papers by Rochelle Forrester include works on Epistemology and the Philosophy of Perception such as Sense Perception and Reality and on quantum mechanics such as the Quantum Measurement Problem, The Problems of Quantum Mechanics and The Bohr and Einstein debate on the meaning of quantum physics. Rochelle Forrester's work is also published on Scribd. Rochelle Forrester is a member of the International Network for Theory of History. 


\begin{abstract}
This paper was written to investigate the order of discoveries made in chemistry leading up to the discovery of the periodic table. New experimental techniques, such as the pneumatic trough, voltaic pile, spectroscopy, and potassium analysis led to the discovery of many new elements and their properties which enabled the discovery of the periodic table. The discoveries led to the demise of the classical theory of the elements, to the end of the phlogiston theory and to the creation of the modern ideas of the elements and of the atomic theory. The paper shows the discoveries were made in a necessary and inevitable order with new experimental techniques leading to the discovery of new elements which eventually led to the discovery of the periodic table.
\end{abstract}

This paper conducts a study of the History of Chemistry from the Phlogiston Theory to the Periodic Table. It traces the changes and new discoveries made in chemistry from the 18 th century to the second half of the 19th century. It examines the chemical revolution of the late 18th century, the discovery of new elements and theories, which eventually led to the development of the periodic table. It concludes with an analysis of the order of the discoveries made in chemistry during that period. It notes that many of the discoveries had to occur in a particular order and that the order of those discoveries was inevitable. This is a conclusion consistent with that stated in my website How Change Happens: A Theory of Philosophy of History, Social Change and Cultural Evolution.

A new scientific attitude had begun to appear in Europe in the 16th and 17th centuries but this had little effect on chemistry until the 18th century. In the 18th century methods for the qualitative and quantitative analysis of minerals improved resulting in the discovery of new compounds and elements. The blowpipe became a common laboratory tool while the practice of weighing precipitated salts was introduced by Torben Bergman when analyzing mineral waters. This practice was improved by Klaproth who heated the salts to dryness before weighing them, which produced more accurate results. Klaproth also began the practice of reporting the actual percentage composition, as produced by his analysis, regardless of whether it totaled $100 \%$ and this allowed the discovery of errors in analysis and led to the discovery of new elements in the materials analyzed.

The new laboratory methods led to the discovery of new elements such as cobalt (1735), platinum (1740-1741), zinc (1746), nickel (1754), bismuth (1757), manganese (1774), molybdenum (1781) tellurium (1782), tungsten (1785) and chromium (1798). The oxides of zirconium, strontium, titanium and yttrium were also discovered. Many of the new substances were metals and this led to the demise of the ancient doctrine of seven metals. In the second half of the 18th century Carl Scheele discovered hydrofluoric acid and the compounds hydrogen cyanide, lactic citric and malic acid and glycerol.

The phlogiston theory was introduced by Becker and Strahl in the late 17th and early 18th centuries. The theory considered all combustible substances contained phlogiston which was lost to the air during the process of combustion. A limited amount of air could only absorb a limited amount of phlogiston, which explained why combustion ceased, if only a limited amount of air was available. Combustion would also cease as soon as substances ran out of phlogiston. The phlogiston released into the air was absorbed by plants, which were eaten by animals, so that the phlogiston was recycled into known combustible materials. 
The main problem with the phlogiston theory is that metals gained weight, when burnt in air, and the theory suggested phlogiston was lost, so one would expect substances to lose weight. This problem became acute, when the gaseous state of matter began to be investigated in the mid 18th century.

It was at this time that gases were becoming much better understood and progress was made on distinguishing compounds from elements. In the early, and mid, 18th century air was considered to be an element. When scientists observed gases with unique properties, their difference from air was assumed to be caused by impurities. Boyle's inverse pressure-volume law also convinced scientists that air was an element since the law applied to all gases. One difficulty with investigating and controlling gases was solved in the early 18th century by Stephen Hales when he invented an apparatus for isolating gases so they could be studied separately. Hales device, known as the pneumatic trough, allowed the collection of gases above water. A bent gun barrel, with its closed end containing various substances, was placed in a fire and with the open end in a vessel of water suspended upside down in a pail of water. The gases released from the substances in the closed end of the gun barrel would collect in the upside down container above the water and separate from the air. Hales apparatus led to the identification of many gases such as carbon dioxide discovered by Joseph Black in 1755; hydrogen discovered by Henry Cavendish in 1766; nitrogen discovered by Daniel Rutherford in 1772; nitrous oxide discovered by Joseph Priestley in 1772 who in the years after that discovered ammonia, sulfur oxide and hydrogen chloride; oxygen was discovered in the 1770's independently by Carl Scheele, Joseph Priestley and Antoine Lavoisier. The ability to isolate, identify and handle gases, soon led to the realization that these gases were forms of matter in the same sense that liquids and solids were.

The study of isolated gases soon showed they were different from each other and the differences resulted from differences in composition rather than from contamination by impurities. The idea of air as an element began to be replaced by the idea of gas as a state of matter.

The phlogiston theory was widely accepted by scientists by the middle of the 18th century. Despite the discovery of oxygen the phlogiston theory continued to be accepted until Lavoisier created a revolution in chemistry which destroyed the phlogiston theory, eliminated the four elements of antiquity and replaced them with the modern concept of elements as substances that could not be broken down and which were the fundamental substances of chemistry. Lavoisier was also involved, in a reform of the nomenclature of chemistry, so that the names of compounds reflected the elements making up the compound.

Many experiments, conducted by Priestley, Lavoisier and others, showed that metals, sulfur and phosphorus, increase in weight when burnt in air. It was also known that when the calx (oxide) produced when these substances were burnt in air, were themselves burnt, using Hale's pneumatic trough, a variety of different airs (gases) would be produced. In particular, experiments were made involving the burning of mercury in air to produce mercury calx, and then the burning of the mercury calx, using the pneumatic trough, to recreate the mercury and a gas in which candles burnt more brightly than in normal air and supported respiration in mice. The air in which the mercury was burnt was not able to support respiration in mice or combustion after the formation of the calx. Measurements made indicated that the weight of the original mercury and air absorbed on burning, equaled that of the calx, and also equaled the weight of the mercury and the gas produced when the calx was burnt.

According to the phlogiston theory the gain in weight of the mercury as it was burnt in the air was caused by the release of phlogiston, which had a negative weight. This explanation was considered by Lavoisier and others, to be absurd. As the increase in weight 
of the mercury equaled the reduction in weight of the air in which the mercury was burnt Lavoisier concluded part of the air had combined with the mercury to form the calx. Equally, as the air produced by burning the mercury calx was different from normal air, and as the air left behind when the mercury was burnt, did not support respiration or combustion, it seemed that a particular constituent part of the air that supported combustion, had been removed from that air and had combined with the mercury to form the calx, and had then been released by the calx into the pneumatic trough. This led Lavoisier to assume that air was not an element, but was composed of several parts, one of which supported combustion and respiration and one that did not. It also led Lavoisier to consider that combustion required the presence of the part of the air that combined with the mercury, and did not involve any release of phlogiston from the substance being burnt. The new gas was eventually called oxygen, by Lavoisier.

A further development involved the burning of hydrogen in air which produced a clear liquid, which on analysis, was shown to be water. Cavendish, Priestley and others, as well as Lavoisier, were involved in these experiments, but Lavoisier was the first to interpret them to mean that water was a compound of oxygen and hydrogen. Lavoisier's interpretation marked the end of the belief, which existed from ancient Greece, that water was an element.

One of the results of the discovery of many different gases after the invention of the pneumatic trough was the understanding of gases as a distinct form of matter. Matter could be seen as changing from a solid to a liquid to a gaseous state, by the application of various degrees of heat.

It also became clear the air, and the gases it was made up of, played a role in chemical reactions. Substances when heated would combine with various gases or would release gases into the atmosphere. In both cases new substances were created by these chemical processes.

A further feature of the discoveries was the confirmation of the law of conservation of mass. Tacitly assumed by many of the chemists, it was confirmed by many experiments dealing with gases combining with metals to form calx, and then the calx, when burnt releasing the gases. When the quantity of gas absorbed by the metal, and the amount released from the calx, are measured, they are found to be the same confirming the scientist's belief in the conservation of mass.

The measurement of substances involved in experiments assumed a much greater role in chemistry, than it had previously. The awareness that metals gained weight when burnt, was an important element in the demise of the phlogiston theory. Chemistry was becoming a quantitative science. Once this occurred the way was open for chemical equations and the calculations of the weight of elements leading to Dalton's atomic theory in chemistry.

Some of Lavoisier's innovations did not survive. His belief that oxygen was a necessary part of all acids and his idea of caloric as an explanation of heat would soon be abandoned. However, by the end of the 18th century, his overall conception was largely adopted throughout Europe.

A debate arose between Berthollet and Proust in the early 19th century as to whether compounds were always formed from fixed proportions of their constituent elements or whether the proportions could vary. Their debate was resolved in favor of fixed proportions although there are now known to be some situations where the constituents of a compound can vary. However in many cases it became clear that compounds were made up of elements that combined in definite and fixed proportions. The question arose as to what lay behind those definite proportions. Dalton showed those proportions were not only fixed but related in a simple numerical manner.

This process was helped by the development of quantitative analysis in chemistry. Before the 19th century most work in chemistry was qualitative and concerned with the 
properties of substances and the courses of particular chemical reactions. By the late 18th century more emphasis was being given to the weight of substances entering into and resulting from chemical reactions.

Lavoisier's concept of elements provided the foundation for Dalton's atomism. Different elements had different atoms and this explained the different properties of the elements. The atomic theory was the outcome of the new quantitative work being done in chemistry, the discovery of fixed proportions in the elements making up compounds and the observation that the proportions were fixed in a particular numerical manner.

The discovery that air is a mixture made up of a number of gases rather than an element raised the question of why was it all mixed together rather than formed in layers with the heaviest gas at the bottom and the lighter gases higher up. Dalton's answer to this problem was the idea that if the particles of a particular kind of gas were self-repulsive but did not repel particles of a different kind of gas, then the formation of layers of gases would not occur. The cause of the repulsion was caloric, Lavoisier's explanation of heat; each particle of gas being surrounded by an atmosphere of caloric. As heat was known to flow from hot substances to colder ones, two equally hot substances would be mutually repellant. The problem remained that all particles of gas had the same repellant (caloric) wouldn't they still repel each other. Dalton considered that the particles of different gases were of different sizes and so would have varying amounts of heat so they would not repel each other. Only particles of the same gas would have the same amount of heat and would repel each other. This theory, although not correct, was the best explanation for the mixing of gases in air before the kinetic theory of gases was developed in the middle of the 19th century. However the idea that the size of particles of different gases would vary, led to the idea that the weight of the particles would vary.

This conclusion was also reached as a result of experiments concerning the solubility of gases in water. It had been observed that the mass of a gas dissolved by a liquid is proportional to the pressure. Elementary gases such as hydrogen and oxygen were less soluble while compound gases such as carbon dioxide were more soluble. Dalton considered the cause of the varying solubilities was the different size of the particles of the different gases. Again the varying size of the particles of different gases led to the idea that the weight of the particles would vary.

Dalton was to call the particles of gases, and of all substances, atoms. Elements were composed of simple atoms and compounds of compounded atoms. The elements varied one from the other, as the atoms making up different elements varied in weight. There were however difficulties in calculating atomic weights. It was impossible to weigh individual atoms so the system of atomic weights had to be based on a comparative system. Dalton chose hydrogen as a base for such a system and gave it an atomic weight of one. The atomic weights of the atoms of other elements were based on how much more they weighed in comparison with hydrogen. To calculate for example how much more oxygen weighed than hydrogen Dalton compared the weight of hydrogen and oxygen making up a quantity of water. He found the oxygen in water weighed 5.5 times as much as the hydrogen (the correct figure is 8) so he assigned an atomic weight of 5.5 to oxygen. Such a system would only work if the number of hydrogen and oxygen atoms in water was known, and in Dalton's time this was not known. To overcome this difficulty Dalton adopted his principle of simplicity when he assumed that if two elements formed only one compound, the compound would consist of one atom of each element. If there were two compounds formed of the same two elements, there would be two atoms of one element and one atom of the other element and so on. As water was the only known compound of hydrogen and oxygen, it was assumed to 
consist of one hydrogen and one oxygen atom. Obviously the principle of simplicity was not a reliable guide to the chemical composition of compounds. The problem of accurate measurement of atomic weights and of accurately assessing the chemical make-up of compounds limited the usefulness and acceptance of the atomic theory. In addition, as the number of elements discovered increased in the early 19th century, it began to look as though there was an increasing number of fundamental particles. Many scientists considered the idea that there were a large number of fundamental particles was absurd.

The atomic theory did obtain support from Gay-Lussac in 1808. Gay-Lussac found that hydrogen combined with oxygen at a ratio of approximately two to one. In other experiments he discovered other gases combine among themselves in simple whole number ratios. This became known as the law of combination of gases. It suggested that equal volumes of different gases contain the same number of particles.

However there were problems with the law of combination of gases by volume. Carbon monoxide, considered to contain one atom of oxygen and one atom of carbon, should be denser than oxygen. Yet, it was known to be less dense than oxygen. A further problem was that one volume of nitrogen combined with one volume of oxygen to give two volumes of nitric oxide rather than the one compound of nitric oxide.

There was considerable confusion over atomic weights, molecular weights and equivalents. This confusion made it impossible to write chemical formulas with confidence. In a chemistry book written by Kekule he quoted nineteen different formulas that had been suggested for acetic acid. Atomic weights in the first half of the 19th century were decided by guesswork and arbitrary rules. Gerhardt corrected Berzelius's atomic weight for sodium and silver by halving them, but he also halved the correct weights for zinc and calcium and so made them incorrect.

A resolution to these problems was offered by Amadeo Avogadro. He distinguished between an atom, as the smallest part of an element which can play a role in a chemical reaction, and a molecule, as the smallest part of a substance. He assumed that molecules of an element could consist of more than one atom of the element. This meant a molecule of hydrogen could contain two atoms of hydrogen. This also meant a molecule could split in two when involved in a chemical reaction. If this happened, then equal volumes of gases could contain the same number of particles. The reaction of nitrogen and oxygen could be explained by two atoms of oxygen joining two atoms of nitrogen to create two molecules of nitric oxide. Avogadro's theory provides for the calculation of molecular weights of substances in the gas or vapor state by determinations of the gas or vapor densities. The determination of densities is made on a relative basis with hydrogen, as the lightest gas, being chosen as the standard. All other substances are expressed as having a weight relative to that of hydrogen. However as the hydrogen molecule consists of two atoms, atomic weights should be related to the weight of half a molecule of hydrogen. Avogadro's theory provided clear information about the number of atoms in a molecule of a compound and provided a firm foundation for the writing of chemical formulas.

However Avogadro's theory was largely ignored. The terminology he used to explain his theory was difficult and many chemists refused to accept that the fundamental particles of elements could contain more than one atom. Avogadro's theory was only adopted after 1860 when Cannizzaro drew chemist's attention to it and explained how it could allow the correct calculation of molecular and atomic weights.

It was only after 1860 that the atomic theory gained considerable acceptance with the acceptance of Avogadro's theory which cleared up problems concerning the atomic weight of elements and the composition of compounds. This was followed by Mendeleev's periodic 
table and finally Einstein's 1905 explanation of Brownian motion as grain pollen being bumped about by the movement of atoms confirmed the atomic theory.

A new method of causing chemical decomposition became available around 1800 . This involved the voltaic pile which allowed a continuous electric current to be passed through a substance causing decomposition. Before 1800 only static electricity had been available for chemical decomposition but the short term nature of the current limited its effectiveness in chemical experiments. The use of the voltaic pile was to allow the discovery of new elements, and showed some substances, previously considered to be elements, were actually compounds. Sir Humphry Davy was to isolate potassium, sodium, barium, strontium and magnesium by means of the voltaic pile while Gay-Lussac and Thenard discovered boron and Courtois discovered iodine. The isolation of potassium led to the discovery of other elements due to the chemical reactivity of potassium. The heating of various compounds with potassium resulted in the discovery of silicon and aluminum.

The use of the voltaic pile led to a further significant discovery. It was observed that when water was decomposed using the voltaic pile, the hydrogen and oxygen formed at different poles. It was then found that when an electric current was passed through solutions of salts, acids formed at the positive pole and bases at the negative pole. This observation led to the development of Berzelius's dualistic theory. Berzelius considered that atoms carried both a positive and negative charge, but only one of the charges was predominant. Metals were electro-positive as they were attracted to the negative pole in electrolysis. Oxygen was the most electro-negative element. As atoms possessed both charges an atom could be negative towards one element and positive towards another. Phosphorus for example was negative towards metals, but positive towards oxygen. This allows a series to be established from the most electro-positive element to the most electro-negative element. Chemical combination happened due to the attraction between opposite electrical charges. When such a combination occurred, the compound formed would be either positive or negative depending on the strength of the charges of the elements making up the compound. If the compound was positive it could then combine with negative compounds and elements and vice versa. Berzelius considered his theory explained the nature of chemical affinity. It would not however be accepted today.

An important new development occurred in 1860 with the development of the spectroscope which allowed the detection of new elements. The color spectrum had been known in Roman times and the refraction of light had been studied by the Arabs, Roger Bacon, Kepler and Descartes. Newton showed that a prism separates white light into its component colors and that another prism could turn the separated colors into white light. The spectrum was extended into the infrared in 1800 by William Herschel and into the ultraviolet in 1801 by W H Williamson.

Dark lines in the spectrum of sunlight passed through a prism were observed by Joseph Fraunhofer in 1814. He studied and mapped the lines (eventually called Fraunhofer lines) and observed similar lines in the spectrum of light from the moon, planets and stars. Fraunhofer also discovered yellow lines in the spectrum of the flame he was using when studying the refractive index of samples of glass. Similar lines were observed in the flames of burning alcohol, oil and tallow when determining refractive indices. Such lines had also been observed in the spectra of many substances by many scientists. They had been observed in the spectra of metallic salts by Thomas Melvill in 1752. David Brewster had observed them in the spectra of "nitrous acid gas", sulphur, iodine vapor and brown oxide of nitrogen. Similar studies were carried out on halogen vapors and other gases. 
The identification of substances by means of the spectrum began when Andreas Marggrat used flame colors to distinguish sodium and potassium salt in 1758. John Herschel showed when the flame colors of boric acid and the chlorides of barium, calcium, strontium and copper were passed through a prism they showed certain lines which could be used to identify the substances. Brewster, after observing sulphur vapor absorbed light from the violet end of the spectrum, and iodine vapor absorbed it from the middle part, suggested "the discovery of a general principle of chemical analysis in which simple and compound bodies might be characterized by their action on definite parts of the spectrum".

This idea was put into practice by the invention of the spectroscope by Bunsen and Kirchoff in 1859. Bunsen used flame colors for the identification of salts in mineral water. Kirchoff suggested better results could be obtained if the light was passed through a glass prism and viewed as a spectrum. Kirchoff also outlined the reason for the bright and dark lines as being emission or absorption lines of light. He set out his laws of spectroscopy as:

1. An incandescent body gives off a continuous spectrum.

2. An excited body gives off a bright-line spectrum.

3. White light passed through a vapour has dark lines where the vapour ordinarily emits light.

The spectroscope provided chemists with an instrument of unprecedented sensitivity for the analysis of chemical substances. The spectroscope was able to map Fraunhofer lines with great accuracy, even when only minute traces of an element were present.

The effects of spectroscopy soon became apparent. Bunsen and Kirchoff discovered cesium in 1860 and rubidium in 1861. Thallium was discovered in 1861 by Crookes and indium in 1863 by Reich and Richter. Spectroscopy was later involved in the discovery of gallium, the rare earths and the rare gases.

When Lavoisier provided the modern definition of an element as a substance that could not be broken down into simpler substances, he provided a list of 33 elements. These included several forms of energy and some substances later found to be compounds. However the attention Lavoisier drew to the elements and new analytical techniques such as the voltaic pile and reaction with potassium, once that element had been isolated by Davy, led to the discovery of many additional elements. Between 1790 and 184431 new elements were discovered but the number of elements remained limited to 58 from 1844 to 1860 as the unknown elements were generally present in minerals in too small quantities to be detected by the analytical techniques available at that time. It took the development of the spectroscope to allow the discovery of new elements to recommence.

The discovery of sufficient elements and the establishment of a reliable system of calculating atomic weights as provided by Avogadro were necessary before the next important development in chemistry. This was the system of classification of the elements known as the periodic table.

The first attempt at such a classification was made by Dobereiner in 1829. Dobereiner observed that it was possible to put the elements into groups of three with the atomic weight of one element in the group being the mathematical average of the other two elements. Dobereiner also observed that the members of the groups all had similar chemical properties.

A number of other attempts were made to discover some sort of relationship between the elements. The most significant of these were made by Beguyer de Chancourtois and John Newlands. Beguyer de Chancourtois in 1862 and 1863 with the benefit of the atomic weights that were accepted after the work of Cannizzaro persuaded chemists to accept Avogadro's 
theory, arranged the elements in order of their increasing atomic weights around a cylinder. Beguyer de Chancourtois pointed out there were remarkable similarities in the elements on the same vertical line on the cylinder.

Newlands, also using Cannizzaro's atomic weights, arranged the elements in order of their atomic weights. He observed that similar elements would appear on a horizontal line if a new column was commenced with each eighth element. In some versions of his table Newlands used blank spaces for unknown elements, but in other versions he did not. Newlands called his arrangement "the law of octaves" but as with Beguyer de Chancourtois, Newlands work was largely ignored.

The idea of the periodic table was accepted due to the work of Mendeleev and Meyer. Mendeleev published his first periodic table in 1869 and a second version was published in 1871. Mendeleev arranged the elements in order of increasing atomic weight and noted the properties of the elements recurred periodically in the table. Gaps were left in the table where Mendeleev considered there were elements yet to be discovered. Using his periodic table Mendeleev predicted the discovery of certain new elements. He stated the approximate atomic weights, valences, the sorts of compounds the element would be found in and other properties the undiscovered elements would have. When the new elements gallium, scandium and germanium were discovered and found to have properties extremely close to those predicted by Mendeleev, his periodic table became widely accepted.

Meyer's periodic table was broadly similar to Mendeleev's but tended to concentrate on the physical properties of the elements, while Mendeleev's concentrated on the chemical properties. Meyer produced a graph in which he plotted the atomic weights against atomic volumes of the elements. The graph showed similar elements appeared at similar places on the curve produced by the graph. Alkali metals appeared at the high points of the curve; non-metals on the ascending sides and metals on the descending sides and in the low points on the curve. Meyer's graph helped to make the periodicity explicable and encouraged acceptance of the periodic table.

The brief foregoing history of chemistry up to the development of the periodic table is provided to show certain aspects of the theory suggested in my book and website $\underline{\text { How }}$ Change Happens: A Theory of Philosophy of History, Social Change and Cultural Evolution. One of those aspects concerns human needs. The needs met by human kinds exploration of the nature of matter was simply the desire to know and understand the universe. Such needs seem to exist in all societies, for example, all societies seem to have creation myths to explain how the universe came into existence. Equally many societies have their own explanations as to the nature of matter. The Babylonians considered all matter was created from a first principle and that first principle was water. Such needs, to explain the nature of matter, would be covered by Maslow's cognitive needs, the need to know, to understand and to explain.

The foregoing history of chemistry is also intended to show how the discoveries made in chemistry were made in a particular order and had to be made in that order. One such discovery was Lavoisier's chemical revolution. This had a number of features such as elements being seen as substances that could not be broken down; air no longer seen as an element; air playing a role in chemical reactions; the concept of a gas being seen as a separate state of matter; confirmation of the law of conservation of mass; explanations of combustion and respiration and a theory of acidity. It seems very apparent that many features of this revolution were dependent upon the discovery of new gases in the mid and late 18th century and in particular upon the discovery of oxygen. It is hardly conceivable that Lavoisier's explanation for combustion and respiration could have been made without the prior discovery 
of oxygen. Lavoisier's experiments with mercury calx showed that part of the air, the part we call oxygen, could cause candles to burn more brightly, but that air without oxygen could not support combustion or respiration in mice. In order to make these discoveries it was necessary for oxygen to be isolated and identified as a particular component of air. Only then could its effect on combustion and respiration be studied and understood. The discovery of oxygen was itself dependent upon a means of isolating and controlling gases as was provided by the pneumatic trough. Without the pneumatic trough Lavoisier would have only been able to perform part of his experiment with mercury calx. He could have burnt the mercury in air to create the calx, an experiment that had been performed many times before. But without the pneumatic trough he could not have burnt the calx in a situation where the oxygen released by the combustion could be controlled and experimented with. The same or similar experiments had been performed by Priestley and Scheele but Lavoisier was the first to interpret the results with a new theory of combustion and respiration. Furthermore, Priestley and Scheele were as dependent on the pneumatic trough as Lavoisier was when it came to the discovery of oxygen. The consequence is that there was an order of discovery from the pneumatic trough to oxygen to Lavoisier's theory of combustion and respiration.

A similar order of discovery was involved in the discovery that air was not an element, that the gases in the air played a role in chemical reactions, and the concept of a gas as a separate state of matter. The discovery of many gases using the pneumatic trough showed air was made up of a number of gases and so was not an element. The role of gases or air in chemical reactions was shown by the gain in weight of metals when burnt in air to produce a calx and then the release of gases when the calx was burnt. The concept of a gas as a separate state of matter was shown when substances could be heated and could be shown to pass through solid, liquid and gaseous states. Again the pneumatic trough played a part in this as substances could be burnt and could be shown to produce particular gases. There was an order of discovery from the pneumatic trough, to the discovery of gases, to the conclusion that air was not an element, and that it plays a role in chemical reactions and that gases are a separate state of matter.

The proof of the law of conservation of mass was dependent upon both the pneumatic trough and upon the increasing use of quantitative studies in chemistry. When metals were burnt, it was known, before Lavoisier; that they increased in weight. However it was only with the use of the pneumatic trough that it was possible to isolate air to show that there was a decrease in the weight of the air that matched the increase in the weight of the metal. The accurate measurement of the decrease in the weight of the air and the increase in the weight of the metal were required before the law of conservation of mass could be confirmed.

The modern concept of an element, as a substance that could not be broken down, was established by Lavoisier, when the traditional elements such as air and water were shown to be made up of simpler substances. Due to the use of the pneumatic trough and the discovery of a number of gases in the mid and late 18th century Lavoisier was able to provide a list of 33 elements, which replaced the traditional elements of earth, fire, air and water. This involved an order of discovery from the pneumatic trough, to the gases discovered through the use of the pneumatic trough to Lavoisier's new concept of elements.

Lavoisier's theories of acids and caloric, while no longer considered correct, were dependent upon the prior discovery of oxygen, which itself was dependent upon the prior discovery of the pneumatic trough. This meant an order of discovery from the pneumatic trough to oxygen to Lavoisier's theories of acid and caloric.

The next major development in chemistry, after Lavoisier's revolution, was Dalton's atomic theory. The atomic theory was based upon Lavoisier's concept of an element. A 
particular element would consist of a particular atom and different elements had different atoms with different weights. It was improvements in quantitative chemistry in the late 18th century, which showed that different elements had different atomic weights. The law of definite and fixed proportions which suggested compounds were made up of elements in definite and fixed proportions and the proportions were related in a simple numerical way provided support for the atomic theory. This was because the atomic theory explained the relationship between the elements as varying in weight in that simple numerical manner. The atomic theory was also based on Dalton's erroneous ideas as to why the different gases in the air did not form layers. Such ideas naturally could only be formed after it had been discovered that the air was made up of a number of different gases. The discovery that air was a mixture was dependent upon the experiments by Lavoisier and others which isolated oxygen, nitrogen and other gases which were dependent on the prior discovery of the pneumatic trough. This means a chain of discoveries runs from the pneumatic trough, to the isolation of oxygen and nitrogen, to the idea of air as a mixture. A further factor in the development of the atomic theory was experiments concerning the solubility of gases in water. These again were dependent on the isolation of various gases by the pneumatic trough, so an order of discovery from the pneumatic trough to the isolation of various gases to the atomic theory can be identified.

The atomic theory could, at the time of Dalton, and eventually did in the 1860's receive support from Gay-Lussac's law of combination of gases which suggests equal volumes of different gases contained the same number of particles. Such a law could only have been proposed when a number of gases had been isolated which required the prior discovery of the pneumatic trough. Yet again an order of discovery can be identified from the pneumatic trough to the discovery of gases, to the law of combination of gases to the atomic theory.

The law of combination of gases naturally led to Avogadro's theory. Avogadro's theory was intended to explain the known behavior of gases during chemical reactions in a manner consistent with the law of combination of gases. This means both the experiments showing the chemical reactions and law of combination of gases were necessarily prior to Avogadro's theory. The acceptance of Avogadro's theory in the 1860's led to an accurate system for calculating atomic weights and to the eventual acceptance of the atomic theory.

The discovery of the voltaic pile led to the discovery of new elements, including potassium, and the discovery of potassium led to the discovery of further elements. The voltaic pile also led to the discovery of the electro-positive to the electro-negative series which in turn led to the development of the dualistic theory. A clear order of discovery runs from the voltaic pile to the discovery of new elements and to the dualistic theory.

The development of the periodic table was dependent upon certain prior discoveries. One was an accurate way of calculating atomic weights, another was the discovery of a sufficient number of elements to allow them to be organized in a coherent table and a further development was sufficient analysis of the properties of the elements to reveal the periodicity of the periodic table. A reliable method of calculating atomic weights was provided by Avogadro's theory. The discovery of the elements occurred throughout the 18th and 19th century assisted by new instruments for the investigation of chemical substances such as the pneumatic trough, the voltaic pile, potassium analysis and the spectroscope. It probably took the invention of the spectroscope in 1859 to allow the discovery of sufficient elements, so a coherent periodic table could be created. The spectroscope itself could only be invented after the discovery of the spectrum, Fraunhofer lines and that Fraunhofer lines allowed the identification of chemical substances. Only when a sufficient number of elements had been discovered and their properties analyzed was it possible to create the periodic table. Early 
attempts to create a coherent table of the elements by Dobereiner, Beguyer de Chancourtois, John Newlands and others had failed due to uncertain atomic weights and an insufficient number of elements being known. It was in 1869 that Mendeleev published his periodic table and 1870 when Meyer published his.

It is of course not surprising there is a specific order of discovery in chemistry from Lavoisier's revolution to the periodic table. Obviously the facts of chemistry were not all discovered at the same time. However, it is contended that the order in which the discoveries were made were, in many cases, inevitable and the discoveries in those cases could not have been made in any other order. In some cases this is obviously so, a good example being the use of the spectroscope to identify elements. It is simply not possible to invent the spectroscope without first discovering the spectrum, then discovering Fraunhofer lines within the spectrum and then the discovery that Fraunhofer lines showed the presence of particular chemical substances. An awareness of all these discoveries was a necessary ingredient to the invention of the spectroscope and all these discoveries had to take place in the order in which they did take place.

A further example would be the development of the periodic table which required certain prior discoveries. The first was the establishment of a coherent definition of an element, distinguishing elements from compounds and mixtures. It was then necessary to create a list of elements and to study their properties, including their atomic weights. Only when a reasonable number of elements had been discovered and their properties had been assessed with a reasonable degree of accuracy was it possible to understand the relationship between the elements and so produce the periodic table.

Yet another example would be Lavoisier's theory of combustion and respiration. It would not have been possible for Lavoisier to come up with such theories without the prior discovery of oxygen.

However other discoveries will take place in a certain order without the later discoveries being dependent upon the earlier discoveries. The dualistic theory was necessarily dependent upon the prior discovery of the electro-positive and electro-negative series and the voltaic pile. It was not however dependent on Lavoisier's chemical revolution and yet it occurred after the chemical revolution. Could the dualistic theory have been created before the chemical revolution? It is possible the dualistic theory could have been invented before the chemical revolution. However such an event was unlikely because discoveries always vary in difficulty; some discoveries will be easier to make than others. The easier discoveries will tend to be made earlier than the more difficult discoveries. This will not always be the case if for example governments and corporations pour resources into a particular area and neglect other areas. Difficult discoveries in areas receiving the resources may well be made before easier discoveries in areas not receiving the resources. The invention of the atomic bomb during World War II and the discoveries made in the space race are such accelerated discoveries caused by governments pushing resources into particular areas. However such situations are unusual and normally easier discoveries will be made before more difficult discoveries.

A further such example would be that Lavoisier's chemical revolution occurred over a hundred years later than Newton's establishment of classical physics. Could Lavoisier's revolution have occurred before Newton's? As Lavoisier's revolution was not dependent upon the earlier revolution in physics it would have been possible for it to have occurred before Newtonian physics. However such a situation would be very unlikely if the discoveries required for Newton's revolution were easier than those required for Lavoisier's. This would seem to be the case as Newton's revolution was substantially dependable on 
directly observable phenomena, the only exception being an accurate understanding of planetary orbits which required the telescope. Lavoisier's revolution was dependent upon phenomena that could not be directly observed such as gases that were discovered by the use of the pneumatic trough. If however, the pneumatic trough had been invented earlier, the chemical revolution could have occurred earlier.

The final result is that there were certain discoveries in chemistry which could not have taken place without certain prior discoveries. These were cases in which the order of discovery was inevitable and no other order of discovery was possible. There were other cases where the order of discovery was not inevitable, but where there was a likely or probable order of discovery as certain discoveries were easier to make than other discoveries, and so were likely to be made earlier than the other discoveries. The degree to which one discovery was easier than another would determine the likelihood of it being made before the other discovery.

Bibliography:

Hudson, J (1992) The History of Chemistry: Chapman \& Hall New York Findlay, A (1965) A Hundred Years of Chemistry (3rd ed): Methuen, London Ihde, A J (1964) The Development of Modern Chemistry: Dover, New York Leicester, H M (1956) The Historical Background of Chemistry: Dover, New York Partington, J R (1961-70) A History of Chemistry: Macmillan, London 